Familias monoparentales y el desarrollo social en los adolescentes

\title{
Familias monoparentales y el desarrollo social en los adolescentes
}

\section{Single-parent families and social development in adolescents}

Famílias monoparentais e desenvolvimento social em adolescentes

Patrícia J. López-Mero I

pattylopez@hotmail.com

Maria C. Pibaque-Tigua II

mary35bebe@hotmail.com

Recibido: 18 de enero de 2018 * Corregido: 19 de mayo de 2018 * Aceptado: 27 de junio de 2018

I. Magister en Gerencia Educativa, Diploma Superior en Intervención Social, Licenciada En Ciencias de la Educación Especialidad Castellano y Literatura, Profesora de Segunda Enseñanza Especialidad Castellano y Literatura, Licenciada en Trabajo Social Especialidad Atención a la Familia y al Desarrollo Comunitario, Universidad Laica Eloy Alfaro de Manabí, Manta, Ecuador.

II. Magister en Educación Parvularia, Trabajadora Social, Licenciada en Trabajo Social, Universidad Laica Eloy Alfaro de Manabí, Manta, Ecuador. 


\section{Resumen}

Los adolescentes se encuentran frente a diversas problemáticas que se ven reflejadas en su contexto social y educativo provenientes del hogar, hijos de familias monoparentales que han cambiado sus conformaciones y dinámicas convirtiéndolas en más complejas. Ante lo expuesto, en este estudio se analizaron las familias monoparentales en el desarrollo social de los hijos adolescentes. Para ello, se aplicó una metodología cualitativa de tipo descriptivo con el fin de captar información sobre las familias monoparentales y el desarrollo social en los hijos adolescentes. El diseño fue no experimental. El instrumento fue una entrevista estructurada. La población la constituyeron familias con características monoparentales y con hijos adolescentes. El análisis de la información fue bajo un enfoque de tipo hermenéutico. Entre sus resultados se pudo observar que los hijos adolescentes presentan problemas en su desarrollo social y entorno familiar, lo que suele reflejarse en el ámbito educativo permitiendo concluir que los hijos que provienen de las familias monoparentales investigadas, tienen poca interacción y participación social.

Palabras clave: familias monoparentales; desarrollo social y adolescentes.

\section{Abstract}

Adolescents face various problems that they are reflected in its social and educational context from home, children of single-parent families that have changed their conformations and dynamics turning them into more complex. Before, lone-parent families in the social development of the teens were analyzed in this study. To do this, applied a descriptive qualitative methodology in order to capture information on single-parent families and the social development in the teens. The design was not experimental. The instrument was a structured interview. Constituted the population families with single-parent characteristics and teenage children. The analysis of the information was under a hermeneutic approach. Among its results could be observed the teens have problems in their social and family environment, which is often reflected in the educational environment allowing conclude that children who come from single-parent families investigated they have little social interaction and social participation.

Keywords: single-parent families; social development and adolescents. 


\section{Resumo}

Os adolescentes se deparam com diversos problemas que se refletem no contexto social e educacional do lar, filhos de famílias monoparentais que mudaram suas conformações e dinâmicas, tornando-as mais complexas. Diante do exposto, neste estudo analisamos famílias monoparentais no desenvolvimento social de crianças adolescentes. Para isso, aplicou-se uma metodologia qualitativa descritiva, a fim de capturar informações sobre famílias monoparentais e desenvolvimento social em crianças adolescentes. $\mathrm{O}$ design não foi experimental. $\mathrm{O}$ instrumento foi uma entrevista estruturada. A população era composta por famílias com características monoparentais e filhos adolescentes. A análise das informações foi baseada em uma abordagem hermenêutica. Dentre os resultados observouse que os adolescentes têm problemas em seu ambiente social e familiar, que muitas vezes se reflete na educação levando à conclusão de que as crianças que vêm de famílias monoparentais pesquisados têm pouca interação e participação social.

Palavras chave: famílias monoparentais; desenvolvimento social e adolescentes.

\section{Introducción}

La familia es la unidad social compuesta por un grupo de personas fusionadas por vínculos consanguíneos, afectivos y que a los efectos de los reglamentos sociales validos debe darse ayuda mutua, distribuir los recursos, tratarse entre sí, proyectar el bien propio, se la considera como una institución parcialmente constante, es la unidad de la sociedad aunque no ha permanecido del todo estática, sobre todo en estos últimos años ha presentado y experimentado transformaciones, donde la familia tradicional quedo pospuesta por nuevas organizaciones familiares lo que ha ocasionado alteraciones en los lazos interpersonales e intrapersonales. La familia evoluciona y así continuará, pero permanecerá ya que es la entidad humana más apropiada en la sociedad como tal es el molde de la identidad y del desarrollo social de quienes la integran.

A nivel mundial la familia monoparental está conformada por madres y padres que no tienen pareja, que están solteros, separados, divorciados o viudos, convirtiéndose en una cifra elevada, la mayoría tienen vivos a sus verdaderos progenitores, por lo general es la madre, constituyendo un hogar monoparental, el padre no vive con sus hijos, pero comparte la patria potestad y la responsabilidad de manutención aun estando ausente del hogar. Evidentemente hay que añadir que en Ecuador todavía podemos apreciar el modelo familiar nuclear que ha constituido el patrón esencial de las familias y 
que es idealizada como un modelo educativo, pero este ha ido perdiendo campo ya que se halla ante una diversidad de modelos familiares por los cambios demográficos, siendo una realidad visible en nuestro contexto el cual está tomando importancia por su incremento y las problemáticas sociales que repercute en aspectos de la vida cotidiana como perder el contacto y el control de los hijos sobre lo que hacen o dejan de hacer, a veces por falta de tiempo no se dialoga con ellos sobre que les preocupa o temas que sean del interés familiar.

La familia está influenciada por el contexto social en donde se incluye y evoluciona, al ajustarse a las influencias se altera en impulsor de cambios, experimentando reorganización en su estructura, su compromiso, sus roles y valores, hoy la familia sigue siendo el habitad originario del individuo, el primer medio y el último refugio, siendo un pilar elemental para la sociedad. Cabe señalar, que los sistemas familiares han atravesado por profundas modificaciones, hogares más minúsculos, incremento de divorcios y de familias monoparentales, ante todas estas modificaciones sociales algunas familias viven inconvenientes a la hora de efectuar sus responsabilidades y cada vez les cuesta más dedicarse a los niños, adolescentes y adultos mayores, así como a impulsar a que las nuevas generaciones aprendan el funcionamiento de la existencia en la sociedad.

Evidentemente hay que añadir que en Ecuador todavía podemos apreciar el modelo familiar nuclear que ha constituido el patrón esencial de las familias y que es idealizada como un modelo educativo, pero este ha ido perdiendo campo, ya que se encuentra ante una diversidad de modelos familiares por los cambios demográficos, siendo una realidad visible en nuestro contexto el cual está tomando importancia por su incremento y las diversas problemáticas sociales que repercute en aspectos de la vida cotidiana como perder el contacto y el control de los hijos sobre lo que hacen o dejan de hacer, a veces por falta de tiempo no se dialoga con ellos sobre qué les preocupa o temas que sean del interés familiar. Ante lo expuesto en este artículo se analizan las familias monoparentales en el desarrollo social en los adolescentes.

\section{Desarrollo}

La familia es la unidad social compuesta por un grupo de personas fusionadas por vínculos consanguíneos, afectivos y que a los efectos de los reglamentos sociales validos debe darse ayuda mutua, distribuir los recursos, tratarse entre sí, proyectar el bien propio, se la considera como una institución parcialmente constante, es la unidad de la sociedad aunque no ha permanecido del todo estática, sobre todo en estos últimos años ha presentado y experimentado transformaciones, donde la 
familia tradicional quedo pospuesta por nuevas organizaciones familiares lo que ha ocasionado alteraciones en los lazos interpersonales e intrapersonales. La familia evoluciona y así continuará, pero permanecerá ya que es la entidad humana más apropiada en la sociedad como tal es el molde de la identidad y del desarrollo social de quienes la integran.

Basado en el criterio de la familia es la unión de personas que comparten un proyecto vital de existencia en común que se supone duradero, en el que se generan fuertes sentimientos de pertenencia a dicho grupo (Barraquel, 2017), la familia siempre será el principal pilar de la sociedad, donde el individuo se origina, educa y desarrolla. Cuando en ella se presentan problemas repercuten inevitablemente en los miembros que integran a esa familia. Según Minuchin, "el rol se define como el elemento que localiza la posición entre los miembros de la familia, se refiere a las pautas de conducta por medio de los cuales la familia asigna funciones necesarias". (Loja, M; Pulla J y López, 2013). De allí que la familia juega una función primordial para el equilibrio, cuidado, estructura de valores, es propulsor y freno de diversos actos, produce orgullo, es motivo de júbilo y melancolía, que forman parte del diario vivir. El rol de la familia es un apoyo donde la persona construye poco a poco las propias características de su personalidad.

Funciones de la familia: El ser humano es un componente de la estructura familiar, la familia como institución cumple varias funciones sociales, entre las más importantes están:

Función biosocial: La familia cumple la función de reproducción ya que en ella se gesta la necesidad de la descendencia y se crean las condiciones para el desarrollo físico, reproductora a psicológico escala familiar y social incide en sus integrantes, en indicadores como la sociedad tales como: densidad poblacional, fecundidad y natalidad.

Función económica: Se ejerce por medio de la realización de actividades para garantizar la integridad de los miembros. Incluye la obtención y administración de los recursos, tanto monetarios como bienes de consumo, la satisfacción de necesidades básicas bienestar de la familia.

Función Educativa-Cultural: Se incluye bajo esta denominación la influencia que ejerce la familia en sus miembros y en todo el proceso de aprendizaje individual y el propio desarrollo familiar. Es la función que produce el proceso de transmisión de experiencias histórico-social en la vida cotidiana. Es también la transmisión de valores ético- morales que se derivan de los principios, normas y regulaciones que se observan y se aprenden en la familia en le mundos de las relaciones intra y extra 
familiar. Incluye la contribución al proceso de formación del desarrollo de la personalidad individual y en general a la formación ideológica de las generaciones.

Función afectiva: Esta es la actividad que realiza la familia, de transmitir el amor que se profesan entre sí sus integrantes. El afecto constituye el vehículo por el cual se ejercen el resto de sus funciones como la función biosocial y educativa. La familia tiene es el más afectivo espacio, muro o puntual de contención que tiene el individuo para tolerar las exigentes provenientes del propio proceso de su desarrollo, de los otros grupos e instituciones y de la vida social en el cual se encuentra inmerso.

\section{Función psicosocial:}

Reproducción y reemplazo poblacional.

Cumplimiento de las necesidades básicas de subsistencia y convivencia familiar.

Satisfacción de necesidades afectivas y red de apoyo social.

Contribución a la formación en el desarrollo de la personalidad individual.

Formación de valores éticos, morales y de conducta social.

Transmisión de experiencias histórico-social de los valores de la cultura. (Camejo, R, 2015).

En base al criterio de "Expresan que la familia funciona de acuerdo con su tipología estructural, y cuando la familia es monoparental, las reglas, los roles, la jerarquía y la comunicación se ajustan para mantener el funcionamiento y adecuarse a la nueva estructura". (Puello, S; Silva, P y Silva S, 2014). Las familias funcionan en base a su estructura, pasando por grandes cambios sociales, las diversas tipologías de familias e inserción de la globalización han generado una ruptura del modelo tradicional de familia nuclear. Según, “Algunos investigadores señalan que...es importante estudiar la función que desempeña el padre o la madre que lidera la familia, para favorecer el desarrollo social de los hijos". (Puello, S; Silva, P y Silva S, 2014). El rol del padre/madre de familia es fundamental en la formación social, cognitiva e integral de los adolescentes, ya que esta aporta a que participen de manera activa y aprendan a negociar sus dificultades y solventar sus necesidades, del mismo modo que aporta a que el adolescente interactúe de modo efectivo con los demás miembros de su entorno familiar y social. 


\section{Tipologías de familia}

\section{Existen diversas estructuras familiares:}

Familia nuclear: Es la familia conviviente formada por los miembros de un único núcleo familiar, el grupo formado por los miembros de una pareja y/o sus hijos. (Forero, 2014)

Familia extensa o conjunta: Incluye a padres, hijos, hermanos de los padres con sus propios hijos, abuelos, tíos abuelos, bisabuelos (generaciones ascendentes). (Forero, 2014)

Familias homoparentales: Una relación estable entre dos personas del mismo sexo. Los hijos llegan por intercambios heterosexuales de uno o ambos miembros de la pareja, adopción y /o procreación asistida. (Córdova, 2012)

Familias con un solo progenitor o monoparentales: "casos de separación, abandono, divorcio, muerte o ausencia por motivos forzosos (trabajo, cárcel, etc. de uno de los padres, el otro se hace cargo de los hijos y conviven" (Córdova, 2012, p78)

Según "La modalidad de madre soltera o divorciada, es la más común en la categoría monoparental" (Forero, 2014, p54)

La mayoría de los hogares monoparentales están liderados por mujeres, donde las responsabilidades y desafíos caen en un solo progenitor sin poder encomendar las funciones al otro, siendo la madre la única encargada de sustentar el hogar y en criar a sus hijos, enfrentando diversidad de problemáticas que se presentan en su estructura familiar. Una de las problemáticas que enfrenta este tipo de familias es "la dimensión estructural o económica, referida a la carencia de recursos materiales que afecta a la subsistencia, provocada por la exclusión del mercado de trabajo" (González, 2014, p.88)

La madre o padre se siente incapaz de poder llevar a cabo solo la crianza de sus hijos por la falta de oportunidades laborables y de apoyo, limitando sus recursos para poder subsistir, disminuyendo su amplitud de adaptación y capacidad para desarrollarse adecuadamente, generando una economía desfavorable y el agotamiento físico y mental por el cuidado que deben brindar a sus hijos, pero por otro lado el progenitor se ve en la necesidad de cumplir sus obligaciones laborales limitando las responsabilidades familiares para llevar el sustento económico, reduciendo el tiempo y actividades dedicadas a sus hijos. 


\section{Familias monoparentales y el desarrollo social en los adolescentes}

\section{Desarrollo social}

Para Barranco y Vargas (2013) lo explican "Como desarrollo social se conoce la evolución y el mejoramiento en las condiciones de vida de los individuos de una sociedad y en las relaciones que estos individuos mantienen entre sí” (p.33). Las habilidades sociales ocupan una función importante en el óptimo desarrollo tanto en la infancia como la adolescencia por lo que permiten desenvolverse y relacionarse de una mejor manera logrando una eficaz socialización, el adolescente mediante su aprendizaje consigue integrarse antes los cambios que acontecen fuera y dentro de él, es un agente de cambio social, no se mantiene inmóvil ante los hechos sociales.

Adolescencia. La adolescencia símbolo de maduración en biológica, social, psíquica en cada individuo, según Jiménez (2016), "En el plano social se realiza una transición del estado de dependencia socioeconómica total a una relativa independencia. “(p.48). En lo social sus relaciones interpersonales con familia y amistades presentan un quiebre, además del impulso de independencia lo que ocasiona conflictos dentro del hogar acompañadas de acciones inadecuadas que necesitan ser corregidas. También, Morales y Limones (2012), expresan "se puede decir que todo lo que rodea a él o la joven... ejercen influencia y al mismo tiempo se ven enfrentados a distintos cambios sociales, estímulos y desafíos que generan respuestas necesarias para su desarrollo personal y social.

\section{Las familias monoparentales en el desarrollo social de los adolescentes}

Los cambios ocurridos en la familia, en las últimas décadas son debidos a la interrelación de diversos factores, implicando que la definición tradicional de esta y las funciones que cada uno de sus miembros juegan dentro de ella se han ido modificando, como es en el caso de las familias monoparentales en su estructura no ejecutan sus funciones provocando un desequilibrio y mal funcionamiento a nivel del sistema familiar. La familia desempeña un rol significativo en la sociedad, pues tiene como encargo social el cuidado y defensa de los hijos, a la vez que constituye la primera institución prestadora de servicios sociales, al responder a las necesidades sociales, educacionales, de salud y protección hacia sus miembros.

Es precisamente en el seno familiar donde se adquieren los primeros hábitos, conductas, costumbres, modos de vida que van fomentando la personalidad del individuo, además en ella se adquieren las primeras experiencias sociales que están vinculadas de modo intenso y durante largo tiempo. (Cobas, 
M y Gamboa D, 2015). La progenie integrada por un solo padre proyecta en sus hijos el sentimiento interno de abandono, resentimiento, baja autoestima, disminuido rendimiento escolar, con tendencia a ser retirados o suspendidos del ámbito escolar, a tener dificultades con sus compañeros y propenso a tener una conducta antisocial, en comparación con los adolescentes que viven con ambos padres que tienen un mejor crecimiento social y emocional.

El apresurado crecimiento de las sociedades acompañado de la pluralidad de las influencias interculturales, han atraído una realidad confusa de valores a los que los y las adolescentes son sensibles, la inestabilidad de su estructura, los cambios demográficos, entre otros llevan a cabo la rebeldía en el joven siendo riesgosas para su desarrollo, las familias monoparentales tienen particularidades de otras tipologías de familia, yendo más allá de su estructura cabe recalcar que comparten muchas problemáticas en común con las otras.

\section{Metodología}

En la presente investigación se aplicó una metodología cualitativa, de tipo descriptivo con el fin de captar información sobre las familias monoparentales y el desarrollo social en los hijos adolescentes. El diseño fue no experimental que según Gómez (2010), corresponde a "representar un escenario que ya viene dado, no obstante, se pueda distinguir valores para evaluar relaciones entre variables" (p.87). El instrumento fue una entrevista estructurada. La población la constituyeron familias con características monoparentales y con hijos adolescentes. El análisis de la información fue bajo un enfoque de tipo hermenéutico.

\section{Análisis y discusión de los resultados}

Posterior a los datos obtenidos del estudio de los hogares monoparentales, fue posible observar que los hijos presentan problemas en su desarrollo social y entorno familiar, lo que suele reflejarse en el ámbito educativo.

Se determinó que la madre aparte de cumplir con el rol de educar, cuidar, proteger, brindar afecto y seguridad a sus hijos le ha tocado desempeñar un papel diferente. La progenitora ante la limitación económica y por la falta de apoyo por parte del padre de sus hijos se ve en la obligación de salir a trabajar y para mantener sola el hogar lo que implica mayor esfuerzo.

Se comprobó que los hijos adolescentes de hogares monoparentales manifiestan en su comportamiento diferentes cambios como poca interacción y participación social, siendo factores negativos que impiden su desarrollo integral. 
Familias monoparentales y el desarrollo social en los adolescentes

Fue posible verificar que la existencia de numerosas familias monoparentales se debe a diferentes situaciones como problemas conyugales, económicos e incomprensión de la pareja entre otros fundamentos, que desencadenan una desestructuración familiar.

\section{Conclusiones}

La madre de los hogares monoparentales visitados, son quienes cumplen de mejor manera su rol para lograr no solo un buen desarrollo de sus hijos, si no atender sus necesidades afectivas y emocionales, considerando el papel de madre como una responsabilidad a tiempo completo.

Los hijos que provienen de las familias monoparentales investigadas, tienen poca interacción social y participación social afectándoles directamente a su rendimiento académico con incidencia en las relaciones interpersonales por su complejidad para ajustarse a un nuevo estilo de vida.

Develaron los hijos algún grado de culpabilidad por la ruptura familiar nuclear que pasaron a ser monoparentales, cuando las verdaderas razones fueron por problemas conyugales, económicos, incomprensión y causas no manifiestas.

Se comprobó que la mayoría de las familias monoparentales investigadas están estructuradas por madre e hijos y por padre e hijos y en la mayoría de los casos, es la mujer quien representa la autoridad principal en el hogar para la formación y educación de sus hijos.

\section{Referencias Bibliográficas}

Gómez, D, y Roquet, J. (2010). Metodología de la investigación. México: Red Tercer Milenio. Revista Publicando, 4 No 10. (1). 2017, 458-474. ISSN 1390, 9304473.

Barranco, A., y Vargas, D. (2013). En Educación Infantil (pág. p. 110). Madrid: CEP S.L.

$\begin{array}{lllllll}\text { Puello S, Silva } & \text { P, } & \& & \text { Silva } & \text { Silva } & \text { (2014). }\end{array}$ https:/www.google.com.ec/search?q=Puello+Scarpati\%2C+Silva+Petruz $\% 2 \mathrm{C}+\% 26+$ Silva+Silva $\%$ 2C+2014\&oq=Puello + Scarpati\%2C + Silva + Petruz $\% 2 C+\% 26+$ Silva + Silv.

$\begin{array}{lllll}\text { Loja } & \text { M } & \text { Pulla, } & \text { J(2013). }\end{array}$ https://www.google.com.ec/search?biw=1366\&bih=635\&ei=JvNgW7jLKIL1 zgLEr4YDQ\&q=Mayra+Fernanda+Loja+prado\%2C+Janeth+Patricia+Pulla+López\%2C+201Barraquel, D. E. (marzo de 2017). Universidad Técnica de Cotopaxi. Obtenido de Problemas familiares que afectan en el rendimiento académico: http://repositorio.utc.edu.ec/bitstream/27000/4003/1/T-UTC-0342.pdf Luch, R (2015). La familia y su estructura. España. Editorial El Cid. 
Forero, N (2014). Calidad de vida laboral y la disposición al cambio organizacional en funcionarios de empresas de la ciudad de Bogotá - Colombia. Universidad Católica de Colombia.www.scielo.org.co/pdf/acp/v19n1/es_v19n1a05.pd.

https://www.google.com.ec/search?ei=7fNgW5mwNo-

SzwKksrHQBw\&q=Forero\%2C+2014\&oq=Forero\%2C+2014\&gs_l=psy-

ab.12...61123.61123.0.63100.1.1.0.0.0.0.2

Córdova, L (2012). Obtenido de Tipologías Familiares: http://trabajosocialj.blogspot.com/2012/07/tipologias-familiares.html

Isabel Lázaro González, 2014. Infancia y protección internacional en Europa. España. Ediciones CEGAL. Jiménez https://www.google.com.ec/search?ei=G_hgW6n7Cc7PzwK96oKADA\&q=libros+de+jimenez+201 6\&oq=libros + de+jimenez+2016\&gs_l=psy-ab.12...4864.7575.0.9441.

Morales, M y Limones, J (2012). Universitos Humanísticas. Uribe Mallarino, Consuelo Desarrollo social y bienestar Universitas Humanística, vol. XXXI, núm. 58, 2004, pp. 11-25 Pontificia Universidad Javeriana Bogotá, Colombia. http://www.redalyc.org/articulo.oa?id=79105802

Cobas, M $\quad$ G Gamboa,

D. https://www.google.com.ec/search?q $=($ Cobas + Moeira $+\% 26+$ Gamboa + Delgado $\% 2 \mathrm{C}+2015 \&$ oq $=(\mathrm{Co}$ bas + Moeira $+\% 26+$ Gamboa + Delgado $\% 2 \mathrm{C}+2015 \&$ aqs $=$ chrome.69i5 . 